\title{
Discrete remnants of orbifolding
}

\author{
Steffen Biermann, ${ }^{1}$ Andreas Mütter $\odot,{ }^{2}$ Erik Parr $\odot,{ }^{2}$ Michael Ratz, ${ }^{3}$ and Patrick K. S. Vaudrevange $\odot^{2}$ \\ ${ }^{1}$ School of Mathematical Sciences, University of Nottingham, Nottingham NG7 2RD, United Kingdom \\ ${ }^{2}$ Physik Department T75, Technische Universität München, James-Franck-Straße, \\ 85748 Garching, Germany \\ ${ }^{3}$ Department of Physics and Astronomy, University of California, Irvine, California 92697-4575, USA
}

(Received 1 July 2019; published 23 September 2019)

\begin{abstract}
We revisit the residual symmetries that survive the orbifold projections, and find additional transformations that have been overlooked in the past. Some of these transformations are outer automorphisms of the downstairs continuous symmetry group. Examples of these transformations include the left-right parity of the Pati-Salam model and its left-right symmetric subgroup.
\end{abstract}

DOI: 10.1103/PhysRevD.100.066030

\section{INTRODUCTION}

Gauge symmetry breaking via orbifolding [1-3] is a popular alternative to spontaneous breakdown of gauge symmetry in four dimensions. There are many reasons for this, including the observation that the infamous doublettriplet splitting problem has a simple solution [4-10]. The low-energy continuous gauge symmetry in these models is well studied [2,9]. The main purpose of this paper is to point out that there are additional discrete symmetries that have not been identified, or discussed, in this context thus far.

This paper is organized as follows. In Sec. II we review some basic facts on orbifolding. In Sec. III we revisit the conditions for residual symmetries and shall show that in the past some symmetries were missed. We illustrate this important fact by a few examples in Sec. IV, i.e., we give one example where a higherdimensional $\mathrm{SO}(10)$ grand unified theory (GUT) is broken by an orbifold to Pati-Salam including left-right parity (also known as $D$-parity). In addition, we present two examples which could be of relevance for flavor model building from orbifold GUTs. Finally, Sec. V contains our summary. Some details are deferred to the Appendixes.

\section{ORBIFOLD GUT BREAKING}

Let us collect some basic facts on orbifolding. For the sake of definiteness we consider six-dimensional settings in which two dimensions get compactified, but our findings

Published by the American Physical Society under the terms of the Creative Commons Attribution 4.0 International license. Further distribution of this work must maintain attribution to the author(s) and the published article's title, journal citation, and DOI. Funded by SCOAP ${ }^{3}$. do not depend on the number of dimensions. Consider a six-dimensional Yang-Mills theory with upstairs gauge group $\mathcal{G}$, where we denote the generators of the Lie algebra in the Cartan-Weyl basis $H_{I}$ and $E_{w}$ collectively by $\mathrm{T}_{a}^{(\mathrm{CW})}$. In a first step, this theory is compactified on a two-torus $\mathbb{T}^{2}$ defined by the lattice vectors $e_{1}$ and $e_{2}$, see Appendix A for more details. We can choose the torus lattice such that it exhibits a $\mathbb{Z}_{N}$ rotational symmetry $\vartheta$ with $\vartheta^{N}=1$, where for $N=3,4,6$ (i.e., the allowed orders $N \neq 2$ of the wallpaper groups in two dimensions) we set $\vartheta e_{1}=e_{2}$, while in the case $N=2$ the basis vectors $e_{1}$ and $e_{2}$ have to be linear independent. In order to orbifold the two-torus $\mathbb{T}^{2}$ to a $\mathbb{T}^{2} / \mathbb{Z}_{N}$ orbifold we mod out this $\mathbb{Z}_{N}$ symmetry, i.e., we identify points $y$ on $\mathbb{T}^{2}$ which are related by a $(360 / N)^{\circ}$ rotation,

$$
y \stackrel{\mathbb{Z}_{N}}{\longmapsto} \vartheta y \sim y
$$

Note that under this geometrical action our six-dimensional fields transform as

$$
\begin{aligned}
V^{\mu}(x, y) & \stackrel{\mathbb{Z}_{N}}{\longmapsto} V^{\mu}\left(x, \vartheta^{-1} y\right), \quad \text { and } \\
\chi(x, y) & \stackrel{\mathbb{Z}_{N}}{\longmapsto} \exp \left(\frac{2 \pi \mathrm{i}}{N}\right) \chi\left(x, \vartheta^{-1} y\right),
\end{aligned}
$$

where the $\chi$ fields transform as the internal components of a six-dimensional vector $V^{M}(x, y)$ of six-dimensional Lorentz symmetry. Moreover, the $\mathbb{Z}_{N}$ orbifold can be extended from its pure geometric action equation (1) to include a discrete $\mathbb{Z}_{N}$ transformation from the gauge symmetry $\mathcal{G}$, i.e.,

$$
\mathrm{T}_{a}^{(\mathrm{CW})} \stackrel{\mathbb{Z}_{N}^{\mathrm{orb}}}{\longmapsto} P \mathrm{~T}_{a}^{(\mathrm{CW})} P^{-1} \quad \text { with } \quad P^{N}=\mathbb{1}
$$


where $P \in \mathcal{G}$ acts as a discrete gauge transformation, ${ }^{1}$ see Eq. (A5) with $U=P=$ constant. Since we restrict ourselves to Abelian orbifolds, we can choose the Cartan generators $H_{I}$ of $\mathcal{G}$ such that $P$ can be expanded as

$$
P=\exp (2 \pi \mathrm{i} V \cdot H),
$$

where the vector $V$ is "quantized" such that $P^{N}=\mathbb{1}$.

\section{A. Orbifold conditions}

Next, in addition to the torus boundary conditions (A4), we impose orbifold boundary conditions

$$
\begin{gathered}
V^{\mu}(x, \vartheta y)=P V^{\mu}(x, y) P^{-1}, \\
\chi(x, \vartheta y)=\exp \left(\frac{2 \pi \mathrm{i}}{N}\right) P \chi(x, y) P^{-1} .
\end{gathered}
$$

Using

$P H_{I} P^{-1}=H_{I} \quad$ and $\quad P E_{w} P^{-1}=\exp (2 \pi \mathrm{i} V \cdot w) E_{w}$, where $w$ denotes the root vector of $E_{w}$, we obtain

$$
V_{I}^{\mu}(x, \vartheta y)=V_{I}^{\mu}(x, y)
$$

$$
V_{w}^{\mu}(x, \vartheta y)=\exp (2 \pi \mathrm{i} V \cdot w) V_{w}^{\mu}(x, y)
$$

$$
\chi_{I}(x, \vartheta y)=\chi_{I}(x, y),
$$

$$
\chi_{w}(x, \vartheta y)=\exp \left(2 \pi \mathrm{i}\left(V \cdot w+\frac{1}{N}\right)\right) \chi_{w}(x, y) .
$$

\section{RESIDUAL GAUGE SYMMETRIES}

We consider the possibility of unbroken discrete symmetries from $\mathcal{G}$. In this case, a symmetry transformation from $\mathcal{G}$ remains unbroken if it commutes with the orbifold boundary condition (5), i.e.,

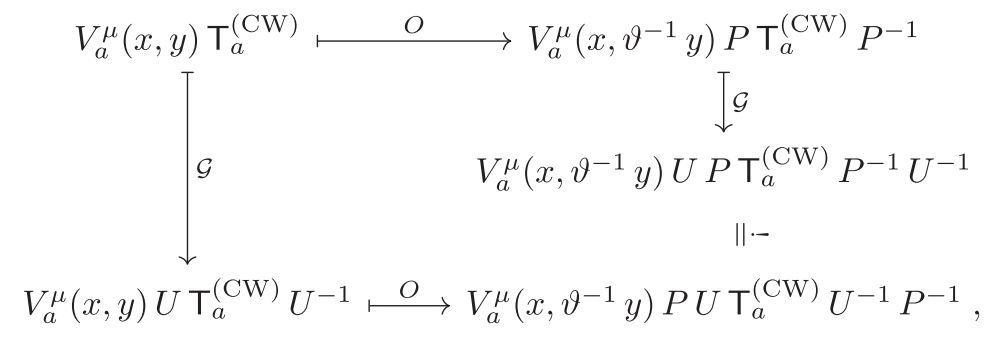

for a global, unbroken transformation $U \in \mathcal{G}$, see Eq. (A5). Consequently, we obtain the condition

$$
\mathrm{T}_{a}^{(\mathrm{CW})}\left(P^{-1} U^{-1} P U\right)=\left(P^{-1} U^{-1} P U\right) \mathrm{T}_{a}^{(\mathrm{CW})}
$$

Due to Schur's lemma, it follows that there is a constant $c \in \mathbb{C}$ such that

$$
P^{-1} U^{-1} P U=:[P, U]=c \mathbb{1},
$$

where we use the definition of the (group-theoretical) commutator of two group elements (as opposed to Lie algebra elements), $[A, B]=A^{-1} B^{-1} A B$ [11]. Using $[A B, C]=B^{-1}[A, C] B[B, C]$ we find for $\ell>1$

\footnotetext{
${ }^{1}$ We ignore the possibility to choose an outer automorphism of $\mathcal{G}$ as gauge action [9]. Furthermore, the order of $P$ can in general differ from the order of $\vartheta$.
}

$$
\begin{aligned}
{\left[P^{\ell}, U\right] } & =\left[P P^{\ell-1}, U\right]=P^{-\ell+1} \underbrace{[P, U]}_{=c \mathbb{1}} P^{\ell-1}\left[P^{\ell-1}, U\right] \\
& =c\left[P^{\ell-1}, U\right] .
\end{aligned}
$$

Consequently, $\left[P^{\ell}, U\right]=c^{\ell} \mathbb{1}$ and for $\ell=N$ we obtain

$$
\mathbb{1}=\left[P^{N}, U\right]=c^{N} \mathbb{1},
$$

using that $P$ is of order $N$ (i.e., $P^{N}=\mathbb{1}$ ), hence, $c^{N}=1$. In summary, our main condition for unbroken symmetries after orbifolding reads

$P^{-1} U^{-1} P U=:[P, U]=\omega^{k} \mathbb{1} \quad$ for $k \in\{0,1, \ldots, N-1\}$,

where $\omega=\mathrm{e}^{\frac{2 \pi i}{N}}$. Since $P, U \in \mathcal{G}$ also $[P, U]$ must be from $\mathcal{G}$. Moreover, $[P, U] \propto \mathbb{1}$. Thus, $[P, U]$ must be from the center of $\mathcal{G}$, i.e.,

$$
\omega^{k} \mathbb{1} \in Z(\mathcal{G}) \quad \text { for some } \quad k \in\{0,1, \ldots, N-1\} .
$$


This constrains the allowed values of $k$. For example, the center of $\mathrm{SU}(M)$ is $\mathbb{Z}_{M}$, while $\omega$ is of order $N$. That is, these additional residual symmetries require the order of the orbifold twist and the dimension of the group center to not be coprime.

\section{A. Unbroken continuous gauge symmetries}

There are two related ways to identify the unbroken gauge symmetries after orbifolding.

First, as is well known, the unbroken gauge interactions are mediated by the zero modes of the gauge bosons. These are the modes with trivial boundary conditions, Eq. (7). Thus, the gauge bosons $V_{I}^{\mu}(x, y)$ and $V_{w}^{\mu}(x, y)$, which are associated to the Cartan generators $H_{I}$ and to those roots $w$ for which $V \cdot w \in \mathbb{Z}$, have trivial boundary conditions and hence massless modes in four dimensions.

Second, we can use our main condition (13) to identify the unbroken continuous symmetries [9]. The unbroken continuous symmetries are continuously connected to the identity $U=\mathbb{1}$. Hence, we have to set $k=0$ in Eq. (13) and expand $U=\exp \left(\mathrm{i} \alpha_{a} \mathrm{~T}_{a}^{(\mathrm{CW})}\right) \approx \mathbb{1}+\mathrm{i} \alpha_{a} \mathrm{~T}_{a}^{(\mathrm{CW})}$. In this way, Eq. (13) yields the condition for a generator of the unbroken gauge symmetry

$$
P^{-1}\left(\alpha_{a} \mathrm{~T}_{a}^{(\mathrm{CW})}\right) P=\left(\alpha_{a} \mathrm{~T}_{a}^{(\mathrm{CW})}\right) .
$$

Since the boundary condition $P$ is expanded in terms of the Cartan generators $H_{I}$, Eq. (4), we can use Eq. (6) to confirm that the Cartan generators $H_{I}$ and the generators $E_{w}$ with $V \cdot w \in \mathbb{Z}$ satisfy Eq. (15), i.e., they remain unbroken after orbifolding.

\section{B. Unbroken discrete gauge symmetries}

In addition to the unbroken continuous gauge symmetries, our main condition (13) can have additional solutions which then lead to further discrete remnants from the higher-dimensional gauge symmetry $\mathcal{G}$. Importantly, these discrete symmetries can originate from our main condition (13) either for $k=0$ (see the example in Sec. IVA) or for $k \neq 0$ (see the examples in Sec. IV B).

Altogether, we confirm that the gauge symmetry breaking pattern of orbifold GUTs discussed in [9] yields the correct unbroken continuous gauge symmetry. However, in order to obtain the full picture of continuous and discrete remnants from orbifolding the discussion needs to be extended. By doing so, we obtain additional symmetries, which have, to our knowledge, not been discussed in the literature. In what follows, we detail this in a few explicit examples.

\section{EXAMPLES AND APPLICATIONS}

In this section, we illustrate our general findings in a few examples.

\section{A. Gauge origin of $\boldsymbol{D}$-parity and left-right parity}

The Pati-Salam model [12] can have, in addition to the continuous gauge group

$$
G_{\mathrm{PS}}=\mathrm{SU}(4) \times \mathrm{SU}(2)_{\mathrm{L}} \times \mathrm{SU}(2)_{\mathrm{R}},
$$

a $\mathbb{Z}_{2}$ symmetry $D$ that exchanges the $\mathrm{SU}(2)$ factors and acts on SU(4) representations as complex conjugation. This symmetry is part of the $\mathrm{SO}(10)$ supergroup containing $G_{\mathrm{PS}}$, and can be preserved in four-dimensional (4D) models of grand unification if one breaks $\mathrm{SO}(10)$ by giving a vacuum expectation value to a 54-plet $[13,14]$. At the level of the left-right symmetric subgroup of $G_{\mathrm{PS}}, G_{\mathrm{LR}}=$ $\mathrm{SU}(3)_{\mathrm{C}} \times \mathrm{SU}(2)_{\mathrm{L}} \times \mathrm{SU}(2)_{\mathrm{R}} \times \mathrm{U}(1)_{B-L}$, this $\mathbb{Z}_{2}$ is the well-known left-right parity [15]. That is, the symmetries of these settings are

$$
\begin{aligned}
& {\left[\mathrm{SU}(4) \times \mathrm{SU}(2)_{\mathrm{L}} \times \mathrm{SU}(2)_{\mathrm{R}}\right] \rtimes \mathbb{Z}_{2} \text { or }} \\
& \quad\left[\mathrm{SU}(3)_{\mathrm{C}} \times \mathrm{SU}(2)_{\mathrm{L}} \times \mathrm{SU}(2)_{\mathrm{R}} \times \mathrm{U}(1)_{B-L}\right] \rtimes \mathbb{Z}_{2} .
\end{aligned}
$$

The purpose of the following discussion is to show that this $\mathbb{Z}_{2}$ factor is a residual symmetry of the corresponding orbifold GUT, which to our knowledge has not been pointed out before.

To this end, consider a theory with $\mathrm{SO}(10)$ symmetry in higher dimensions compactified on a $\mathbb{Z}_{2}$ orbifold such as $\mathbb{S}^{1} / \mathbb{Z}_{2}$ or $\mathbb{T}^{2} / \mathbb{Z}_{2}$. We choose the GUT breaking boundary condition

$$
P_{\mathrm{PS}}=\operatorname{diag}\left(-\mathbb{1}_{6} ; \mathbb{1}_{4}\right)
$$

As is well known, the continuous low-energy gauge symmetry is $G_{\mathrm{PS}}$ [10]. However, there is an additional $\mathbb{Z}_{2}$ symmetry.

In more detail, our main condition (13) yields

$$
\left[P_{\mathrm{PS}}, U_{(k)}\right]=(-1)^{k} \mathbb{1} \quad \text { for } \quad k \in\{0,1\},
$$

and we search for the unbroken elements $U_{(k)} \in \mathrm{SO}(10)$. For $k=0$ condition (19) reads

$$
P_{\mathrm{PS}} U_{(0)}=U_{(0)} P_{\mathrm{PS}} .
$$

The most general $\mathrm{SO}(10)$ matrix satisfying this condition reads

$$
U_{(0)}=\left(\begin{array}{cc}
O_{6} & 0 \\
0 & O_{4}
\end{array}\right) \in \mathrm{SO}(10) .
$$

Consequently, we find the conditions

$$
\begin{aligned}
O_{6}^{T} O_{6} & =\mathbb{1}_{6} \quad \text { and } \quad O_{4}^{T} O_{4}=\mathbb{1}_{4} \quad \text { and } \\
\operatorname{det} O_{6} & =\operatorname{det} O_{4}= \pm 1
\end{aligned}
$$


Hence, $U_{(0)}$ with $\operatorname{det} O_{6}=\operatorname{det} O_{4}=+1$ yields

$$
\begin{aligned}
& O_{6} \in \mathrm{SO}(6) \simeq \mathrm{SU}(4) \quad \text { and } \\
& O_{4} \in \mathrm{SO}(4) \simeq \mathrm{SU}(2)_{\mathrm{L}} \times \mathrm{SU}(2)_{\mathrm{R}},
\end{aligned}
$$

while $U_{(0)}$ with $\operatorname{det} O_{6}=\operatorname{det} O_{4}=-1$ can be generated by

$$
\begin{aligned}
& O_{6}=\operatorname{diag}(1,1,1,1,1,-1) O_{6}^{\prime} \text { and } \\
& O_{4}=\operatorname{diag}(1,1,1,-1) O_{4}^{\prime},
\end{aligned}
$$

for $O_{6}^{\prime} \in \mathrm{SO}(6) \simeq \mathrm{SU}(4)$ and $O_{4}^{\prime} \in \mathrm{SO}(4) \simeq \mathrm{SU}(2)_{\mathrm{L}} \times$ $\mathrm{SU}(2)_{\mathrm{R}} \cdot{ }^{2}$ Let us remark that setting $k=1$ in our main condition (19) does not yield further unbroken symmetries.

Consequently, the $\mathbb{Z}_{2}$ orbifold boundary condition $P_{\mathrm{PS}}$ breaks $\mathrm{SO}(10)$ down to

$$
G_{\mathrm{PS}}=\left(\mathrm{SU}(4) \times \mathrm{SU}(2)_{\mathrm{L}} \times \mathrm{SU}(2)_{\mathrm{R}}\right) \rtimes \mathbb{Z}_{2},
$$

where the generator of the additional $\mathbb{Z}_{2}$ remnant symmetry can be chosen to be

$$
D=\operatorname{diag}(-1,1,1,1,1,1 ; 1,-1,-1,-1) .
$$

Here, we write $D$ in this suggestive way because this will make it very obvious how this $\mathbb{Z}_{2}$ acts. We could have represented it by any diagonal matrix with entries \pm 1 subject to the condition that the number of -1 's on either side of the semicolon is odd.

How does this $\mathbb{Z}_{2}$ act on representations? Consider first the $\mathrm{SO}(4)$ subblock. There, the transformation $D$ can be understood by analogy to parity acting on spinors $(1 / 2,0) \oplus$ $(0,1 / 2)$ of $\mathrm{SU}(2) \times \mathrm{SU}(2)$ in $4 \mathrm{D}$ Euclidean space-time: parity interchanges the $\mathrm{SU}(2)$ representations. Translated to Pati-Salam, $D$ acts on $\left(\boldsymbol{r}_{\mathrm{L}}, \boldsymbol{r}_{\mathrm{R}}\right)$ of $\mathrm{SU}(2)_{\mathrm{L}} \times \mathrm{SU}(2)_{\mathrm{R}}$ as

$$
\left(\boldsymbol{r}_{\mathrm{L}}, \boldsymbol{r}_{\mathrm{R}}\right) \stackrel{D}{\longmapsto}\left(\boldsymbol{r}_{\mathrm{R}}, \boldsymbol{r}_{\mathrm{L}}\right),
$$

see also Appendix B for an explicit computation showing how $D$ acts on $\mathrm{SU}(2)_{\mathrm{L}} \times \mathrm{SU}(2)_{\mathrm{R}}$. Similarly, $D$ acts on the $\mathrm{SO}(6) \simeq \mathrm{SU}(4)$ subgroup in analogy to (a Euclidean version of) time reversal, so for any SU(4) representation $\boldsymbol{r}_{4}$

$$
\boldsymbol{r}_{4} \stackrel{D}{\longmapsto} \overline{\boldsymbol{r}}_{4} .
$$

Altogether a representation $\left(\boldsymbol{r}_{4}, \boldsymbol{r}_{\mathrm{L}}, \boldsymbol{r}_{\mathrm{R}}\right)$ of $\mathrm{SU}(4) \times$ $\mathrm{SU}(2)_{\mathrm{L}} \times \mathrm{SU}(2)_{\mathrm{R}}$ transforms under $D$ as

$$
\left(\boldsymbol{r}_{4}, \boldsymbol{r}_{\mathrm{L}}, \boldsymbol{r}_{\mathrm{R}}\right) \stackrel{D}{\longmapsto}\left(\overline{\boldsymbol{r}}_{4}, \boldsymbol{r}_{\mathrm{R}}, \boldsymbol{r}_{\mathrm{L}}\right) .
$$

So this $\mathbb{Z}_{2}$ exchanges $(\mathbf{4}, \mathbf{2}, \mathbf{1})$ and $(\overline{\mathbf{4}}, \mathbf{1}, \mathbf{2})$, i.e., the leftand right-handed fermions of the standard model. That is,

\footnotetext{
${ }^{2}$ Note that " $\simeq$ " means "up to $\mathbb{Z}_{2}$ factors," but these $\mathbb{Z}_{2}$ 's are different from the one we are going to discuss next.
}

this simple orbifold GUT gives rise to the well-known leftright parity [15], where it originates from $\mathrm{SO}(10)$ and is hence clearly a discrete gauge symmetry. Ironically, the representation of its generator (26) supports the naming in [15], where this transformation has been called parity. Even though it is not the ordinary space-time transformation that gets broken spontaneously there, as the left-right symmetric model is chiral and even in its unbroken phase does not preserve parity, this transformation does act on the $\mathrm{SO}(6) \simeq$ $\mathrm{SU}(4)$ and $\mathrm{SO}(4) \simeq \mathrm{SU}(2)_{\mathrm{L}} \times \mathrm{SU}(2)_{\mathrm{R}}$ representations in an analogous way as space-time parity does.

Altogether we have found that the breaking pattern of the $\mathrm{SO}(10)$ orbifold GUT is

$\mathrm{SO}(10) \stackrel{\mathbb{Z}_{2} \text { orbifold }}{\longrightarrow}\left[\mathrm{SU}(4) \times \mathrm{SU}(2)_{\mathrm{L}} \times \mathrm{SU}(2)_{\mathrm{R}}\right] \rtimes \mathbb{Z}_{2}$,

where $\mathbb{Z}_{2}$ corresponds to the left-right parity and is in particular a nontrivial outer automorphism of $G_{\mathrm{PS}}$. It is amusing to see that the same mechanism that breaks the gauge symmetry and provides us with a solution to the doublet-triplet splitting problem automatically leads to this symmetry.

This parity has a simple geometric interpretation in terms of root lattices, which already can be obtained from a lower-dimensional example. Consider the breaking of $\mathrm{SO}(5)$ to $\mathrm{SO}(4)$ with a twist $P_{5}=\operatorname{diag}(1,-1,-1,-1,-1) \in$ $\mathrm{SO}(5)$. This breaking removes a simple root from the root lattice (see Fig. 1), and the simple roots of $\mathfrak{g} \mathfrak{u}(2)_{\mathrm{L}} \oplus$ $\mathfrak{S} \mathfrak{t}(2)_{\mathrm{R}}$ span a sublattice of the original $\mathfrak{g} \mathfrak{o}(5)$ lattice. However, the Weyl reflection with respect to the plane orthogonal to the "broken" $\operatorname{root} \alpha_{(2)}^{\mathfrak{g o}(5)}$ is a symmetry of the $\mathfrak{s} \mathfrak{t}(2)_{\mathrm{L}} \oplus \mathfrak{g} \mathfrak{t}(2)_{\mathrm{R}}$ sublattice, and exchanges (the generators of) the $\mathfrak{g} \mathfrak{i}(2)$ algebras.

The analogous statement holds in the full Pati-Salam example, but depicting the transformation $D$ as a Weyl reflection is more difficult since the rank of $\mathfrak{g} \mathfrak{o}(10)$ is 5 . As we shall see, the residual transformations in the examples in Sec. IV B can also be related to elements of the Weyl group.

Discussing the phenomenological implications of this symmetry is beyond the scope of this work, we only note the revived interest in this transformation in [16] and references therein.

\section{B. Non-Abelian residual symmetries}

In what follows, we present two examples in which the higher-dimensional gauge group gets broken by the orbifold to a semidirect product of an Abelian gauge symmetry with a discrete $\mathbb{Z}_{N}$ factor. Such symmetries naturally contain non-Abelian discrete groups that can be used as flavor symmetries.

\section{1. $\mathbb{T}^{2} / \mathbb{Z}_{4}$ Orbifold GUT}

We choose a six-dimensional gauge symmetry $\mathcal{G}=$ $\mathrm{SU}(2)$ and $\left|e_{1}\right|=\left|e_{2}\right|$ with $e_{1} \cdot e_{2}=0$. This lattice has a 

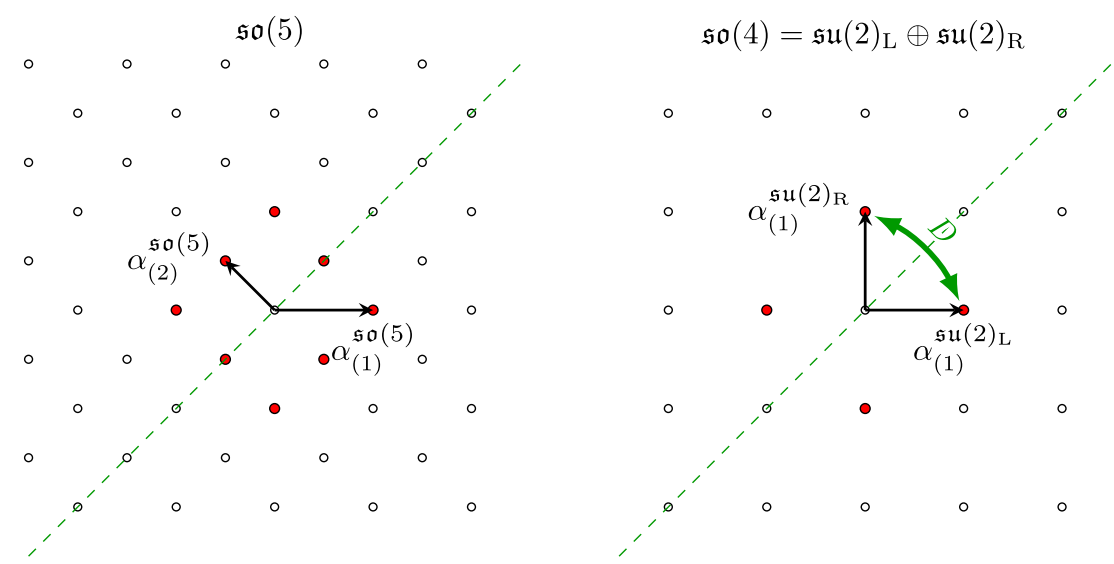

FIG. 1. Root lattices of $\mathfrak{g} \mathfrak{v}(5)$ and its $\mathfrak{g} \mathfrak{v}(4)=\mathfrak{g} \mathfrak{t}(2)_{\mathrm{L}} \oplus \mathfrak{G} \mathfrak{t}(2)_{\mathrm{R}}$ subalgebra. The roots of the respective algebras are depicted by filled circles and the simple roots are given by arrows. A Weyl reflection with respect to the plane perpendicular to $\alpha_{(2)}^{\mathfrak{s o}(5)}$ exchanges the generators of the two $\mathfrak{g} \mathfrak{t}(2)$ algebras. Hence, the outer automorphism $D$ of $\mathfrak{g} \mathfrak{t}(2)_{\mathrm{L}} \oplus \mathfrak{g} \mathfrak{t}(2)_{\mathrm{R}}$ is generated by the Weyl reflection at the broken $\operatorname{root} \alpha_{(2)}^{\mathfrak{s} \mathfrak{o}(5)}$ of $\mathfrak{g} \mathfrak{v}(5)$

$\mathbb{Z}_{4}$ rotational symmetry $\vartheta$ that we divide out in order to construct a $\mathbb{T}^{2} / \mathbb{Z}_{4}$ orbifold. The associated gauge embed$\operatorname{ding} P$ is chosen as

$$
P=\left(\begin{array}{cc}
\mathrm{i} & 0 \\
0 & -\mathrm{i}
\end{array}\right) \in \mathrm{SU}(2) \quad \text { where } \quad P^{4}=\mathbb{1}
$$

Then, the unbroken symmetry is given by those $U_{(k)} \in$ $\mathrm{SU}(2)$ that satisfy

$\left[P, U_{(k)}\right]=\exp \left(\frac{2 \pi \mathrm{i} k}{4}\right) \mathbb{1} \quad$ where $\quad k \in\{0,1,2,3\}$.

Since $P, U_{(k)} \in \mathrm{SU}(2)$, the right-hand side of Eq. (32) has to be an element of $\mathrm{SU}(2)$, too. Moreover $\left[P, U_{(k)}\right] \propto \mathbb{1}$, thus, it has to be from the center $Z(\mathrm{SU}(2))=\mathbb{Z}_{2}$. Consequently, Eq. (32) can only have solutions for $k \in\{0,2\}$.

To find all solutions of Eq. (32) we parametrize a general element $U_{(k)} \in \mathrm{SU}(2)$ using $p, q \in \mathbb{C}$ as

$$
\begin{aligned}
U_{(k)}= & \left(\begin{array}{cc}
p & q \\
-\bar{q} & \bar{p}
\end{array}\right) \in \mathrm{SU}(2) \\
& \text { where } \quad \operatorname{det}\left(U_{(k)}\right)=|p|^{2}+|q|^{2}=1 .
\end{aligned}
$$

Then, Eq. (32) reads

$$
\left[P, U_{(k)}\right]=\left(\begin{array}{cc}
|p|^{2}-|q|^{2} & 2 \bar{p} q \\
-2 p \bar{q} & |p|^{2}-|q|^{2}
\end{array}\right) \stackrel{!}{=} \exp \left(\frac{2 \pi \mathrm{i} k}{4}\right) \mathbb{1},
$$

which is equivalent to

$$
|p|^{2}-|q|^{2} \stackrel{!}{=} \exp \left(\frac{2 \pi \mathrm{i} k}{4}\right) \quad \text { and } \quad \bar{p} q \stackrel{!}{=} 0
$$

Now, since $|p|^{2}-|q|^{2} \in \mathbb{R}$ we see explicitly that Eq. (34) has no solutions for $k \in\{1,3\}$.

Setting $k=0$ in Eq. (35) we find the unbroken gauge symmetry given by $|p|^{2}=1$ (hence, $p=\mathrm{e}^{\mathrm{i} \alpha}$ ) and $q=0$, i.e.,

$$
U_{(0)}=U_{(0)}(\alpha)=\left(\begin{array}{cc}
\mathrm{e}^{\mathrm{i} \alpha} & 0 \\
0 & \mathrm{e}^{-\mathrm{i} \alpha}
\end{array}\right) \in \mathrm{SU}(2),
$$

where $\alpha \in[0,2 \pi)$. This yields an unbroken $\mathrm{U}(1)$ gauge symmetry. On the other hand, setting $k=2$ in Eq. (35) yields $p=0$ and $|q|^{2}=1$ (thus, $q=\mathrm{ie}^{\mathrm{i} \alpha}$, where the additional factor " $i$ " has been introduced for later convenience), i.e.,

$U_{(2)}=\left(\begin{array}{cc}0 & \mathrm{ie}^{\mathrm{i} \alpha} \\ \mathrm{ie}^{-\mathrm{i} \alpha} & 0\end{array}\right)=\left(\begin{array}{cc}\mathrm{e}^{\mathrm{i} \alpha} & 0 \\ 0 & \mathrm{e}^{-\mathrm{i} \alpha}\end{array}\right)\left(\begin{array}{cc}0 & \mathrm{i} \\ \mathrm{i} & 0\end{array}\right) \in \mathrm{SU}(2)$,

where $\alpha \in[0,2 \pi)$.

Consequently, the unbroken symmetry of $\mathrm{SU}(2)$ is generated by a $\mathrm{U}(1)$ and a $\mathbb{Z}_{4}$, i.e.,

$U_{(0)}(\alpha)=\left(\begin{array}{cc}\mathrm{e}^{\mathrm{i} \alpha} & 0 \\ 0 & \mathrm{e}^{-\mathrm{i} \alpha}\end{array}\right)$ and $U_{(2)}=\left(\begin{array}{cc}0 & \mathrm{i} \\ \mathrm{i} & 0\end{array}\right)$,

where $\left(U_{(2)}\right)^{2}=-\mathbb{1}=U_{(0)}(\pi) \in \mathrm{U}(1)$. The $\mathbb{Z}_{4}$ transformation $U_{(2)}$ acts on the gauge bosons as $\mathbb{Z}_{2}$, i.e.,

$$
V_{a}^{\mu}(x, y) \mathrm{T}_{a}^{(\mathrm{CW})} \longmapsto V_{a}^{\mu}(x, y) U_{(2)} \mathrm{T}_{a}^{(\mathrm{CW})} U_{(2)}^{-1},
$$


see the diagram (8). By explicitly choosing the CartanWeyl basis $H=\frac{1}{\sqrt{2}} \sigma_{3}$ and $E_{ \pm}=\frac{1}{2}\left(\sigma_{1} \pm \mathrm{i} \sigma_{2}\right)$, one verifies that $U_{(2)}$ in Eq. (39) can be understood as the action of the unbroken element $w$ of the Weyl group of $\mathfrak{g} \mathfrak{u}(2)$, i.e.,

$$
w:\left(\begin{array}{c}
H \\
E_{+} \\
E_{-}
\end{array}\right) \longmapsto\left(\begin{array}{c}
-H \\
E_{-} \\
E_{+}
\end{array}\right) .
$$

In summary, the six-dimensional SU(2) gauge symmetry is broken by this $\mathbb{Z}_{4}$ orbifold according to

$$
\mathrm{SU}(2) \stackrel{\mathbb{Z}_{4}^{\mathrm{orb}}}{\longmapsto}\left(\mathrm{U}(1) \rtimes \mathbb{Z}_{4}\right) / \mathbb{Z}_{2}
$$

Let us remark that this unbroken symmetry after orbifolding contains, for example, the binary dihedral groups $Q_{N}$ with $N=$ even as subgroups [17], including the quaternion group for $N=4$.

\section{2. $\mathbb{T}^{2} / \mathbb{Z}_{3}$ Orbifold GUT}

Next, we choose a six-dimensional gauge symmetry $\mathcal{G}=$ $\mathrm{SU}(3)$ and $\left|e_{1}\right|=\left|e_{2}\right|$ with $e_{1} \cdot e_{2}=-\left|e_{1}\right|^{2} / 2$. This lattice has a $\mathbb{Z}_{3}$ rotational symmetry $\vartheta$ that we divide out in order to construct a $\mathbb{T}^{2} / \mathbb{Z}_{3}$ orbifold. The associated gauge embedding $P$ is chosen as

$P=\left(\begin{array}{ccc}\omega & 0 & 0 \\ 0 & \omega^{2} & 0 \\ 0 & 0 & 1\end{array}\right) \in \mathrm{SU}(3)$ where $P^{3}=\mathbb{1}$,

where $\omega=\exp 2 \pi \mathrm{i} / 3$. Then, the unbroken symmetry is given by those $U_{(k)} \in \mathrm{SU}(3)$ that satisfy

$\left[P, U_{(k)}\right]=\exp \left(\frac{2 \pi \mathrm{i} k}{3}\right) \mathbb{1} \quad$ where $\quad k \in\{0,1,2\}$.

Since $P, U_{(k)} \in \mathrm{SU}(3)$, the right-hand side of Eq. (43) has to be an element of $\mathrm{SU}(3)$, too. Moreover, $\left[P, U_{(k)}\right] \propto \mathbb{1}$, thus, it has to be from the center $Z(\mathrm{SU}(3))=\mathbb{Z}_{3}$. Consequently, Eq. (43) can have solutions for all cases $k \in\{0,1,2\}$.

The unbroken symmetry can be generated by two U(1) factors

$$
U_{(0)}=\left(\begin{array}{ccc}
\mathrm{e}^{\mathrm{i}(\alpha+\beta)} & 0 & 0 \\
0 & \mathrm{e}^{\mathrm{i}(\alpha-\beta)} & 0 \\
0 & 0 & \mathrm{e}^{-2 \mathrm{i} \alpha}
\end{array}\right) \in \mathrm{SU}(3)
$$

and two discrete transformations

$$
\begin{aligned}
& U_{(1)}=\left(\begin{array}{lll}
0 & 0 & 1 \\
1 & 0 & 0 \\
0 & 1 & 0
\end{array}\right) \in \operatorname{SU}(3), \\
& U_{(2)}=\left(\begin{array}{lll}
0 & 1 & 0 \\
0 & 0 & 1 \\
1 & 0 & 0
\end{array}\right) \in \operatorname{SU}(3),
\end{aligned}
$$

where $U_{(2)}=\left(U_{(1)}\right)^{2}$. Since $\left(U_{(1)}\right)^{3}=\mathbb{1}, U_{(1)}$ generates an unbroken $\mathbb{Z}_{3}$. Consequently, the six-dimensional $\mathrm{SU}(3)$ gauge symmetry is broken by the $\mathbb{Z}_{3}$ orbifold according to (see also $[18,19])$

$$
\mathrm{SU}(3) \stackrel{\mathbb{Z}_{3}^{\text {orb. }}}{\longmapsto}[\mathrm{U}(1) \times \mathrm{U}(1)] \rtimes \mathbb{Z}_{3} .
$$

Again, the $\mathbb{Z}_{3}$ can be understood as a remnant of the Weyl group: if we denote the Weyl reflection with respect to the root $\alpha$ by $w_{\alpha}$, conjugating with $U_{(1)}$ has the same action on the generators as the Weyl transformation $w_{\alpha_{(1)}} w_{\alpha_{(2)}}$, where $\alpha_{(I)}, I=1,2$, denote the simple roots of SU(3). The U(1) factors emerge from the standard gauge symmetry breaking by orbifold boundary conditions to the commuting subgroup, see, e.g., Eq. (6) in [9]. However, to our knowledge, there is no systematic way in the previous literature how to derive the (noncommuting) $\mathbb{Z}_{3}$ factor. We also note that if one breaks the $\mathrm{U}(1)$ factors down to $\mathbb{Z}_{3}$ symmetries, this leaves us with $\left(\mathbb{Z}_{3} \times \mathbb{Z}_{3}\right) \rtimes \mathbb{Z}_{3}$, which is known as $\Delta(27)$ and has been proposed as a flavor symmetry.

\section{SUMMARY}

We discussed how gauged discrete symmetries emerge from orbifolds. Although we used the field-theoretic constructions the discussion is purely group theoretical and applies to string-derived orbifolds as well. We identified residual discrete symmetries. These included the so-called left-right parity of the Pati-Salam model or its left-right symmetric subgroup, which, to the best of our knowledge, have been overlooked in the literature so far. These symmetries are inner automorphisms of the upstairs symmetry group but outer automorphisms of the orbifolded setup. Notably, we found that these symmetries do not have to commute with the orbifold twist. Rather, the transformations $U$ have to fulfill the weaker condition

$$
P^{-1} U^{-1} P U=\omega^{k} \mathbb{1} \in Z(\mathcal{G}),
$$

where $P$ is the orbifold twist and $Z(\mathcal{G})$ the center of the group $\mathcal{G}$. In accordance with the usual expectations, all these symmetries are gauged, i.e., local.

\section{ACKNOWLEDGMENTS}

We would like to thank K.S. Babu for useful discussions. This work is supported by the Deutsche 
Forschungsgemeinschaft (SFB1258). The work of M. R. is supported by NSF Grant No. PHY-1719438.

\section{APPENDIX A: TORUS COMPACTIFICATION AND SYMMETRIES}

In six dimensions we assume a Yang-Mills theory with upstairs gauge group $\mathcal{G}$. Then, the standard Lagrangian for the associated gauge bosons $V^{M}(x, y), M=0, \ldots, 5$, reads

$$
\mathcal{L}=-\frac{1}{2} \operatorname{tr}\left(F_{M N} F^{M N}\right),
$$

where $F_{M N}$ denotes the field strength tensor. We expand $V^{M}(x, y)$ in terms of the generators of the Lie algebra of $\mathcal{G}$ in the Cartan-Weyl basis, i.e.,

$$
\begin{aligned}
V^{M}(x, y) & =\sum_{I} V_{I}^{M}(x, y) H_{I}+\sum_{w \in W} V_{w}^{M}(x, y) E_{w} \\
& =\sum_{a} V_{a}^{M}(x, y) \mathrm{T}_{a}^{(\mathrm{CW})}
\end{aligned}
$$

where the index $I$ runs over all Cartan generators $H_{I}, W$ denotes the set of nontrivial roots of $\mathcal{G}$ and we denote all Cartan-Weyl generators collectively by $\mathrm{T}_{a}^{(\mathrm{CW})}$.

An orbifold compactification of this model can be thought of as two steps: first we compactify two dimensions on a two-torus $\mathbb{T}^{2}$ with coordinates $y=\left(y_{1}, y_{2}\right)^{T}$ and then (as described in Sec. II) on a $\mathbb{T}^{2} / \mathbb{Z}_{N}$ orbifold. To do so, we split the gauge fields $V^{M}(x, y)$ into components with index $M=\mu$ in Minkowski space-time and with index $M=4,5$ in the internal two-torus. From a four-dimensional perspective, the fields

$$
V^{\mu} \quad \text { and } \quad \chi=\frac{1}{\sqrt{2}}\left(V^{4}+\mathrm{i} V^{5}\right)
$$

give rise to the gauge bosons of $\mathcal{G}$ and complex scalars, respectively, both transforming in the adjoint of $\mathcal{G}$.

\section{Torus compactification}

We impose boundary conditions on the fields $V_{a}^{\mu}(x, y)$ and $\chi_{a}(x, y)$ compactified on a two-torus $\mathbb{T}^{2}$. To do so, we choose two linearly independent lattice vectors $e_{1}$ and $e_{2}$ that span the torus lattice. Depending on the orbifold, we will choose different torus metrics $G_{i j}=e_{i} \cdot e_{j}$. We take a general, integral linear combination $n_{i} e_{i}$ for $n_{i} \in \mathbb{Z}$, where summation over $i=1,2$ is implied. Torus periodicity implies that for all $n_{i} \in \mathbb{Z}$

$$
\begin{aligned}
& V_{a}^{\mu}\left(x, y+n_{i} e_{i}\right)=V_{a}^{\mu}(x, y), \\
& \chi_{a}\left(x, y+n_{i} e_{i}\right)=\chi_{a}(x, y) .
\end{aligned}
$$

This choice of boundary conditions corresponds to the case of a torus with trivial gauge background fields (i.e., without Wilson lines). Since they are periodic in $y$, the usual Kaluza-Klein reduction yields massless modes for both $V_{a}^{\mu}(x, y)$ and $\chi_{a}(x, y)$ from the four-dimensional point of view. Consequently, the upstairs gauge symmetry $\mathcal{G}$ remains unbroken after torus compactification, i.e.,

$$
\begin{gathered}
V^{\mu} \stackrel{\mathcal{G}}{\longmapsto} U V^{\mu} U^{-1}-\frac{\mathrm{i}}{g}\left(\partial^{\mu} U\right) U^{-1}, \\
\chi \stackrel{\mathcal{G}}{\longmapsto} U \chi U^{-1},
\end{gathered}
$$

with $U=U(x)$ in the fundamental representation of $\mathcal{G}$ and $g$ denoting the associated gauge coupling.

\section{APPENDIX B: D-PARITY IN PATI-SALAM FROM ORBIFOLDING}

In this Appendix, we give an explicit example how one can compute the action of a residual symmetry transformation on the unbroken gauge symmetry. To do so, we consider $D$-parity from the Pati-Salam example in Sec. IVA and work out the consequences of this $\mathbb{Z}_{2}$ on $\mathrm{SO}(4)$. The $\mathfrak{g} \mathfrak{p}(4)$ algebra is generated by six antisymmetric matrices that fulfill

$$
\begin{aligned}
{\left[M_{i}, M_{j}\right] } & =\mathrm{i} \varepsilon_{i j k} M_{k}, \quad\left[N_{i}, N_{j}\right]=\mathrm{i} \varepsilon_{i j k} M_{k}, \\
{\left[M_{i}, N_{j}\right] } & =\mathrm{i} \varepsilon_{i j k} N_{k} .
\end{aligned}
$$

An explicit representation can be chosen as

$$
\begin{array}{ll}
M_{1}=\left(\begin{array}{cccc}
0 & 0 & 0 & 0 \\
0 & 0 & -\mathrm{i} & 0 \\
0 & \mathrm{i} & 0 & 0 \\
0 & 0 & 0 & 0
\end{array}\right), & M_{2}=\left(\begin{array}{cccc}
0 & -\mathrm{i} & 0 & 0 \\
0 & 0 & \mathrm{i} & 0 \\
0 & 0 & 0 & 0 \\
-\mathrm{i} & 0 & 0 & 0 \\
0 & 0 & 0 & 0
\end{array}\right), \\
M_{3}=\left(\begin{array}{cccc}
\mathrm{i} & 0 & 0 & 0 \\
0 & 0 & 0 & 0 \\
0 & 0 & 0 & 0
\end{array}\right), \\
N_{1}=\left(\begin{array}{cccc}
0 & 0 & 0 & -\mathrm{i} \\
0 & 0 & 0 & 0 \\
0 & 0 & 0 & 0 \\
\mathrm{i} & 0 & 0 & 0
\end{array}\right), \\
N_{3}=\left(\begin{array}{cccc}
0 & 0 & 0 & 0 \\
0 & 0 & 0 & 0 \\
0 & 0 & 0 & -\mathrm{i} \\
0 & 0 & \mathrm{i} & 0
\end{array}\right) .
\end{array}
$$


These generators can be "disentangled" by making a basis change $W_{i}^{ \pm}:=\frac{1}{2}\left(M_{i} \pm N_{i}\right)$, for $i=1,2,3$, such that we arrive at the relations

$$
\begin{array}{ll}
{\left[W_{i}^{+}, W_{j}^{+}\right]} & =\mathrm{i} \varepsilon_{i j k} W_{k}^{+}, \quad\left[W_{i}^{-}, W_{j}^{-}\right]=\mathrm{i} \varepsilon_{i j k} W_{k}^{-}, \\
{\left[W_{i}^{+}, W_{j}^{-}\right]} & =0 .
\end{array}
$$

Hence, we have separated the $\mathfrak{g} \mathfrak{v}(4)$ into $\mathfrak{g} \mathfrak{u}(2)_{\mathrm{L}} \oplus \mathfrak{g} \mathfrak{u}(2)_{\mathrm{R}}$. Now, we take $U_{\mathbb{Z}_{2}}=\operatorname{diag}(1,1,1,-1)$, see Eq. (24).
Following the diagram (8), an explicit calculation reveals that a discrete gauge transformation with $U_{\mathbb{Z}_{2}}$ acts as

$$
\begin{aligned}
& W_{i}^{+} \longmapsto U_{\mathbb{Z}_{2}} W_{i}^{+} U_{\mathbb{Z}_{2}}^{-1}=W_{i}^{-}, \\
& W_{i}^{-} \longmapsto U_{\mathbb{Z}_{2}} W_{i}^{-} U_{\mathbb{Z}_{2}}^{-1}=W_{i}^{+} .
\end{aligned}
$$

Hence, we see explicitly that $U_{\mathbb{Z}_{2}}$ interchanges $\mathfrak{S} \mathfrak{i t}(2)_{\mathrm{L}}$ and $\mathfrak{s} \mathfrak{t}(2)_{\mathrm{R}}$.
[1] L. J. Dixon, J. A. Harvey, C. Vafa, and E. Witten, Nucl. Phys. B261, 678 (1985).

[2] L. J. Dixon, J. A. Harvey, C. Vafa, and E. Witten, Nucl. Phys. B274, 285 (1986).

[3] L. E. Ibáñez, J. E. Kim, H. P. Nilles, and F. Quevedo, Phys. Lett. B 191, 282 (1987).

[4] Y. Kawamura, Prog. Theor. Phys. 103, 613 (2000).

[5] R. Barbieri, L. J. Hall, and Y. Nomura, Phys. Rev. D 63, 105007 (2001).

[6] Y. Kawamura, Prog. Theor. Phys. 105, 999 (2001).

[7] G. Altarelli and F. Feruglio, Phys. Lett. B 511, 257 (2001).

[8] A. Hebecker and J. March-Russell, Nucl. Phys. B613, 3 (2001).

[9] A. Hebecker and J. March-Russell, Nucl. Phys. B625, 128 (2002).

[10] T. Asaka, W. Buchmüller, and L. Covi, Phys. Lett. B 523, 199 (2001).
[11] P. Ramond, Group Theory: A Physicist's Survey (Cambridge University Press, Cambridge, England, 2010).

[12] J. C. Pati and A. Salam, Phys. Rev. D 10, 275 (1974); 11, 703(E) (1975).

[13] T. W. B. Kibble, G. Lazarides, and Q. Shafi, Phys. Rev. D 26, 435 (1982).

[14] D. Chang, R. N. Mohapatra, and M. K. Parida, Phys. Rev. Lett. 52, 1072 (1984).

[15] R. N. Mohapatra and G. Senjanovic, Phys. Rev. Lett. 44, 912 (1980).

[16] L. J. Hall and K. Harigaya, arXiv:1905.12722.

[17] H. Ishimori, T. Kobayashi, H. Ohki, Y. Shimizu, H. Okada, and M. Tanimoto, Prog. Theor. Phys. Suppl. 183, 1 (2010).

[18] F. Beye, T. Kobayashi, and S. Kuwakino, Phys. Lett. B 736, 433 (2014).

[19] F. Beye, T. Kobayashi, and S. Kuwakino, J. High Energy Phys. 03 (2015) 153. 